\title{
Determination of pressure in the near-ground space pile terminated and broadening of the surface
}

\author{
Natalia Kupchikova* \\ Astrakhan State University of Architecture and Civil Engineering, department of of construction \\ expertise, 414056, Astrakhan, Tatishchevstr.,18, Russia
}

\begin{abstract}
The article deals with the problem of determining the stress state of a complex pile structure with end broadening in the form of a sphere in the soil mass in the analytical form by a discrete method. The calculation schemes for determining the stress tensor at the boundary of the pile of square and circular cross-section with expansions in the soil massif are shown. The elements of the polynomial are found by the discrete method in rectangular and spherical coordinates, which is a cumbersome complex mathematical apparatus for a modern design engineer. The stresses are determined. At present, as the analysis has shown, the solution of complex geotechnical problems of soil bases and foundations for different types of loads in numerical modeling is carried out using modern software. Numerical modeling and calculation with the help of specialized software systems allows to consider the system "building-foundationground foundation", as dynamic, integrally developing. However, the interaction of the components of this system requires a theoretical justification of the resistance of foundations in the ground environment, especially in complex engineering-geological conditions.
\end{abstract}

\section{Introduction}

The transfer of force from the pile with a broadening to the base causes a stress-strain state in the surrounding circumferential space, while the shape of the outlines and the values of the stress diagrams are determined not only by the magnitude of this effort, but also by the size and geometry of the pile structure itself and the broadening itself.

The analysis of experimental and numerical studies makes it possible to form a calculation scheme for the interaction of the pile with the terminal broadening with the base under the action of a vertical load (Fig. 1).

The determination of pressure in the near-ground massif and their distribution along the lateral surface along the vertical axis and around the broadening can be determined, considering the equilibrium condition for a segment of a pile of elementary length $d z$ when it interacts with the surrounding soil along a contact surface of radius $a$ and diameter $2 a$.

\footnotetext{
Corresponding author: kupchikova79@mail.ru
} 


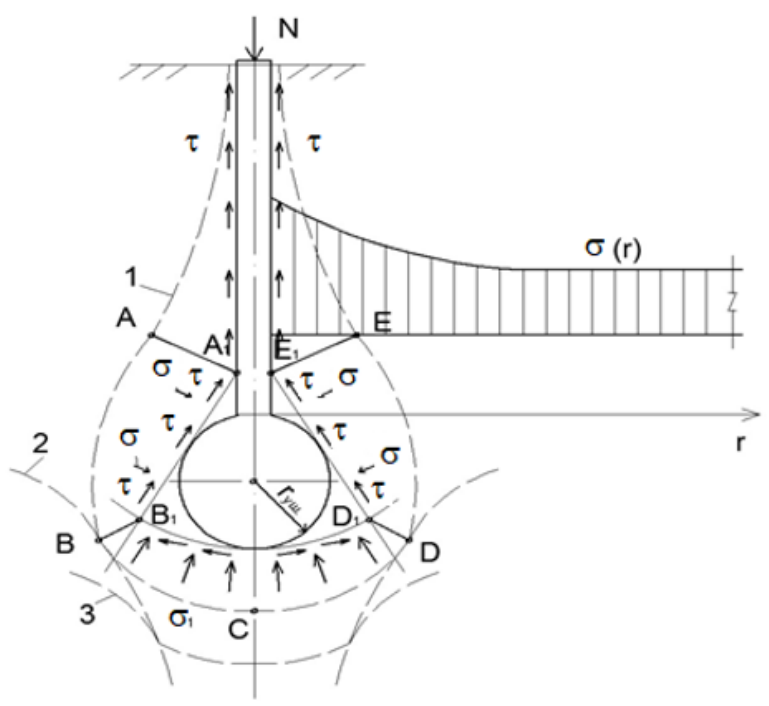

Fig. 1. The design scheme of the stress-strain state of a pile with end broadening

In engineering structures and laboratory studies, consider the continuous arrays of structural bodies and ground media with various inhomogeneous properties of the problem with regard to their stress-strain state using contact problems in a discrete setting.

The design scheme is the broadening of the spherical outline can be represented as follows. Draw the tangents along the lateral surfaces of the terminal broadening until it intersects with the lateral faces of the pile at points $A_{1}$ and $E_{1}$ and the ball surface below the lower edge of the broadening at points $B_{1}$ and $D_{1}$.

The total surface of the limiting state around the pile with the end widening below the bottom of the pile is limited by the ball surface $A B C D E$ formed by the intersection of the isolines 1 and 2 . The distance from the center of gravity of the broadening (the point $O$ ) to the center of the $\operatorname{arc} B D$ (the point $C$ ) is equal to $R$.

\section{Methods}

We use the sequence of the solution by a discrete method (according to Vinokurov L.P. [1]), which is a solution of contact problems for determining the stress-strain state of a foundation in a soil massif, where the desired function is represented in finite-difference form for all variables except one, which is determined by the analytical method, i.e. discretely. The representation of the required function in a discrete form for all variables except one is related to dividing the body into a series of elements and establishing the lines for which the function is found in analytical form.

The constructions of piles with terminal broadenings are compound arrays consisting of prismatic, cylindrical and spherical bodies. The discrete method for compound arrays (piles with broadenings), based on the theory of elasticity and plasticity, combines the idea of a mixed bed, i.e. takes into account the properties of the base with linear proportionality and in the form of an elastoplastic base.

The formulation of differential equations using a discrete method for solving spatial problems that are solved in rectangular and spherical coordinates proceeds as follows: 
- the investigated array (structure) is separated by a system of mutually perpendicular planes parallel to the coordinate planes $x y y z$, by a series of elementary parallelepipeds 1,2 , $3 \ldots n$ of dimensions $a, d, h$. Differential equations for the determination of the functions $f$ (z) expressing the variation of $u, v, w$ with respect to the height $h$ of each of the vertical edges of elementary parallelepipeds are obtained using the equations of the solid body, compiled using the displacement method, which, however, allows satisfying the conditions on the contour given not only in displacements, but also in pressure.

Consider the four adjacent parallelepipeds shown in the plan in Fig. 2 and one arbitrarily taken in the axonometry. Within each group of four separately taken adjacent elementary parallelepipeds having one common central edge $b$, the components of the displacements of the points $u, v, w$ of these parallelepipeds are expressed by power functions of the type (1) of $x$ and $y$, the coefficients of which are certain continuous functions of $f(z)$.

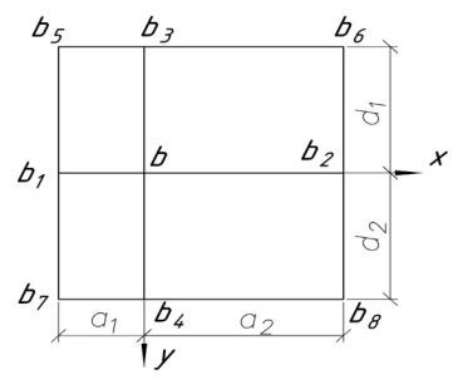

Fig. 2. Parallelepiped system

We denote the vertical edges of parallelepipeds by one letter $b$ for multiplicity. For the components of the displacement of the points of the vertical edges of the articulation of these arrays.

In Fig. 3. two calculation schemes are presented in a discrete method for solving the spatial and contact problems of the structures of a printed and finished prismatic pile with end widening in the form of a sphere. The structure of the pile is represented as an array consisting of a volumetric cylinder - the body of the pile and the sphere - the terminal broadening. The structure of the finished pile can be represented as an array of a volume prism - the pile bodies and also the spherical terminal broadening.

The values of the displacements $u, v, w$ of the edges of elementary prisms, which are functions of $z$, are taken as independent unknowns. In this case, $u$ is the radial displacement, $v$ is the tangential displacement, $w$ is the longitudinal displacement along the $z$ axis.

For $u, v, w$ within the limits of the four contiguous parallelepipeds under consideration, at any level $z$ we take, assuming the origin of the edge $b$, write the nine-term polynomial of the function in which the coefficients $c, d$, and $e$ are functions of $z$ :

$$
\begin{aligned}
& u=c_{0}+c_{1} x+c_{2} y+c_{3} x y+c_{4} x^{2}+c_{5} y^{2}+c_{6} x^{2} y^{2}+c_{7} x y^{2}+c_{8} x^{2} y \\
& v=d_{0}+d_{1} x+d_{2} y+d_{3} x y+d_{4} x^{2}+d_{5} y^{2}+d_{6} x^{2} y^{2}+d_{7} x y^{2}+d_{8} x^{2} y \\
& w=e_{0}+e_{1} x+e_{2} y+e_{3} x y+e_{4} x^{2}+e_{5} y^{2}+e_{6} x^{2} y^{2}+e_{7} x y^{2}+e_{8} x^{2} y
\end{aligned}
$$




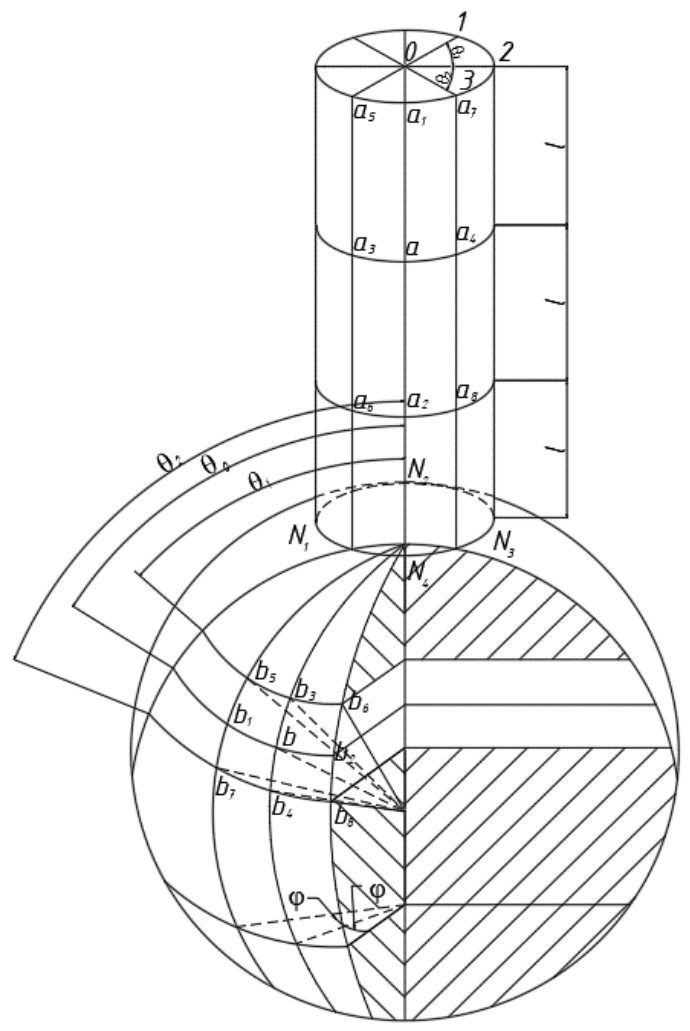

a)

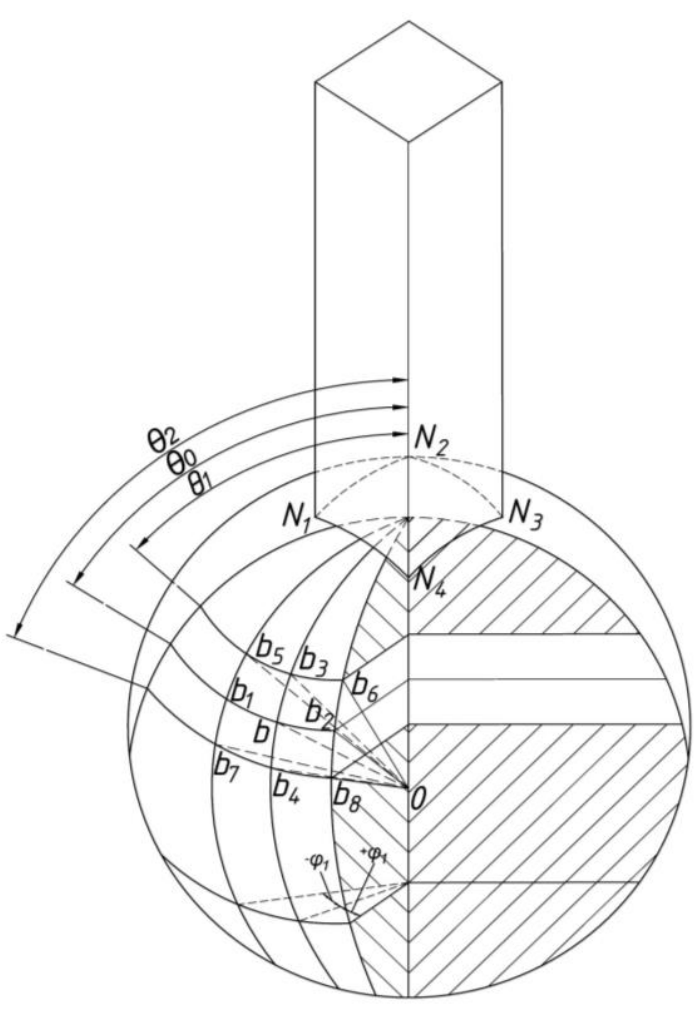

b)

Fig. 3. Estimated scheme in a discrete method of solving the spatial and contact problems of the design of a printed and finished prismatic pile with end widening $(a)$ in the form of a sphere $(b)$

The coefficients $c, d$, and $e$ are determined as a result of solving equations composed of the coincidence conditions at any level $z$ of displacements $u, v, w$ with displacements $u_{\mathrm{b}}, v_{\mathrm{b}}$, $w_{\mathrm{b}} ; u_{\mathrm{b} 1}, v_{\mathrm{b} 1}, w_{\mathrm{b} 1 \ldots \ldots .} u_{\mathrm{b} 8}, v_{\mathrm{b} 8}, w_{\mathrm{b} 8}$ of the points of the nine vertical edges of the four adjacent elementary parallelepipeds under consideration.

The results of approximating the components of displacements $u, v, w$ with respect to the coordinate planes $x y$ yz by polynomials of the type (1) obviously will lessen the divergence from the real ones, the smaller the sizes of $a$ and $d$ elementary parallelepipeds in comparison with the dimensions of $l_{x}$ and $l_{y}$ of the body.

As a result of solving the equations resulting from these conditions, we obtain the following coefficients $c, d$, and $e$. 


$$
\begin{aligned}
& c_{0}=\frac{1}{2}\left[-2\left(\alpha^{2}-1\right) u_{b}+\alpha(\alpha+1) u_{b_{3}}+\alpha(\alpha-1) u_{b_{4}}\right] \\
& c_{1}=\frac{1}{2 \bar{\lambda}}\left[4 \alpha u_{b}-2(\alpha+1) u_{b_{3}}-\alpha(2 \alpha-1) u_{b_{4}}\right] \\
& c_{2}=\frac{1}{4 \varphi_{1}}\left[-2\left(\alpha^{2}-1\right)\left(u_{b_{2}}-u_{b_{1}}\right)+\alpha(\alpha+1)\left(u_{b_{6}}-u_{b_{5}}\right)+\alpha(\alpha-1)\left(u_{b_{8}}-u_{b_{7}}\right)\right] \\
& c_{3}=\frac{1}{4 \varphi_{1} \bar{\lambda}}\left[4 \alpha\left(u_{b_{2}}-u_{b_{1}}\right)-(2 \alpha+1)\left(u_{b_{6}}-u_{b_{5}}\right)-(2 \alpha-1)\left(u_{b_{8}}-u_{b_{7}}\right)\right] \\
& c_{4}=\frac{1}{2 \bar{\lambda}^{2}}\left[-2 u_{b}+u_{b_{3}}+u_{b_{4}}\right] \\
& c_{5}=\frac{1}{4 \varphi_{1}^{2}}\left[-2\left(\alpha^{2}-1\right)\left(-2 u_{b}+u_{b_{1}}+u_{b_{2}}\right)+\alpha(\alpha+1)\left(u_{b_{6}}-u_{b_{5}}\right)-\left(-2 u_{b 3}+u_{b_{5}}+u_{b_{6}}\right)+\right] \\
& c_{6}=\frac{1}{4 \varphi_{1}{ }^{2} \lambda^{2}}\left[\begin{array}{l}
\left.-2\left(-2 u_{b}+u_{b_{1}}+u_{b_{2}}\right)+\left(-2 u_{b_{3}}+u_{b_{5}}+u_{b_{6}}\right)+u_{b_{8}}\right) \\
+\left(-2 u_{b_{4}}+u_{b_{7}}+u_{b_{8}}\right)
\end{array}\right] \\
& c_{8}=\frac{1}{4 \varphi_{1} \lambda^{2}}\left[-2\left(u_{m_{2}}-u_{m_{1}}\right)+\left(u_{m_{6}}-u_{m_{5}}\right)+\left(u_{b_{8}}-u_{b_{7}}\right)\right] \\
& c_{7}=\frac{1}{4 \varphi_{1}{ }^{2} \lambda}\left[\begin{array}{l}
4 \alpha\left(-2 u_{b}+u_{b_{1}}+u_{b_{2}}\right)-(2 \alpha+1)\left(-2 u_{b_{3}}+u_{b_{5}}+u_{b_{6}}\right)- \\
-(2 \alpha-1)\left(-2 u_{b_{4}}+u_{b_{7}}+u_{b_{8}}\right)
\end{array}\right] \\
& \left.c_{h}\right)
\end{aligned}
$$

where

$$
\alpha=\frac{\theta_{0}}{\bar{\lambda}}, \theta_{1}=\theta_{0}-\lambda, \theta_{2}=\theta_{0}+\lambda
$$$$
\alpha_{v} \frac{d^{2} u_{m}}{d r^{2}}+\frac{2 \alpha_{v}}{d r} \frac{d u_{b}}{d r}-\frac{2 \alpha_{v}}{r_{0}^{2}} u_{m}+\frac{\operatorname{ctg} \theta_{0} \beta_{v}}{r_{0}} \frac{d w_{b}}{d r}-\frac{\operatorname{ctg} \theta_{0} \gamma_{v}}{r_{0}^{2}} w_{b}+\frac{\alpha\left(\rho_{v}+\frac{\theta_{0}}{2}\right)}{2 r_{0} \theta_{0}} \frac{d}{d r}\left(w_{b 4}-w_{b_{3}}\right)+
$$$$
+\frac{\beta_{v}}{2 r_{0} \varphi_{1} \sin \theta_{0}} \frac{d}{d r}\left(v_{b_{2}}-v_{b_{1}}\right)-\frac{\alpha\left(\frac{\theta_{0}}{2}+\alpha_{v}\right)}{2 r_{0}^{2} \theta_{0}}\left(w_{b_{4}}-w_{b_{3}}\right)-\frac{\gamma_{v}}{2 r_{0}^{2} \varphi_{1} \sin \theta_{0}}\left(v_{b_{2}}-v_{b_{1}}\right)+
$$$$
+\frac{\alpha \operatorname{ctg} \theta_{0}}{4 r_{0}^{2} \theta_{0}}\left(u_{b_{4}}-u_{b_{3}}\right)+\frac{1}{2 r_{0}^{2} \varphi_{1}^{2} \sin ^{2} \theta_{0}}\left(-2 u_{b}+u_{b_{1}}+u_{b_{2}}\right)+\frac{\alpha^{2}}{2 r_{0}^{2} \theta_{0}}\left(-2 u_{b}+u_{b_{3}}+u_{b_{4}}\right)=0
$$

$\frac{1}{2} \frac{d^{2} v_{m}}{d r^{2}}+\frac{1}{r_{0}} \frac{d v_{m}}{d r}-\frac{1}{r_{0}^{2} \sin ^{2} \theta_{0}} v_{m}+\frac{\beta_{v}}{2 r_{0} \varphi_{1} \sin \theta_{0}} \frac{d}{d r}\left(u_{b_{2}}-u_{b_{1}}\right)+$

$$
+\frac{\alpha_{v}}{\varphi_{1} r_{0}^{2} \sin \theta_{0}}\left(u_{b_{2}}-u_{b_{1}}\right)+\frac{\alpha_{v}}{r_{0}^{2} \varphi_{1} \sin ^{2} \theta_{0}}\left(-2 v_{b}+v_{b_{1}}+v_{m_{2}}\right)+\frac{\alpha c t g \theta_{0}}{4 r_{0}^{2} \theta_{0}}\left(v_{b_{4}}-v_{b_{3}}\right)=0
$$

These equations, containing functions $u, v, w$ of one variable $r$, have variable coefficients. They are transformed into differential equations with constant coefficients by substitution $r=\mathrm{e}^{\mathrm{t}}$. 


$$
\begin{aligned}
& \alpha_{v} \frac{d^{2} u_{b}}{d t^{2}}+2 \alpha_{v} \frac{d u_{b}}{d t}-2 \alpha_{v} u_{b}+\frac{\alpha c t g \theta_{0}}{4 \theta_{0}}\left(u_{b 4}-u_{b 3}\right)+ \\
& +\frac{1}{2 \varphi_{1}^{2} \sin ^{2} \theta_{0}}\left(-2 u_{b}+u_{b_{1}}+u_{b 2}\right)+\frac{\alpha^{2}}{2 \theta_{0}}\left(-2 u_{b}+u_{b_{3}}+u_{b_{4}}\right)+ \\
& +\frac{\beta_{v}}{2 \varphi_{1} \sin \theta_{0}} \frac{d}{d t}\left(v_{b_{2}}-v_{b_{1}}\right)-\frac{\gamma_{v}}{2 \varphi_{1} \sin \theta_{0}} \frac{d}{d t}\left(v_{b_{2}}-v_{b_{1}}\right)+ \\
& +\frac{\alpha\left(\frac{\theta_{0}}{2}+\rho_{v}\right)}{2 \theta_{0}} \frac{d}{d t}\left(w_{b_{4}}-w_{b_{3}}\right)+\beta_{v} \operatorname{ctg} \theta_{0} \frac{d w_{b}}{d t}-\gamma_{v} \operatorname{ctg} \theta_{0} w_{b}- \\
& -\frac{\alpha\left(\frac{\theta_{0}}{2}+\alpha_{v}\right)}{2 \theta_{0}}\left(w_{b_{4}}-w_{b_{3}}\right)=0 \\
& \frac{1}{2} \frac{d^{2} v_{b}}{d t^{2}}+\frac{d v_{b}}{d t}-\frac{1}{\sin ^{2} \theta_{0}} v_{b}+\frac{\alpha c t g \theta_{0}}{4 \theta_{0}}\left(v_{b_{4}}-v_{b_{3}}\right)+\frac{\alpha_{v}}{\varphi^{2}{ }_{1} \sin \theta_{0}} \times \\
& \times\left(-2 v_{b}+v_{b_{1}}+v_{b_{2}}\right)+\frac{\alpha^{2}}{2 \theta_{0}^{2}}\left(-2 v_{b}+v_{b_{3}}+v_{b_{4}}\right)+\frac{\beta_{v}}{2 \varphi_{1} \sin \theta_{0}} \frac{d}{d t}\left(u_{b_{2}}-u_{b_{1}}\right) \\
& +\frac{\alpha_{v}}{\varphi_{1} \sin \theta_{0}}\left(u_{b 2}-u_{b 1}\right)+\frac{\operatorname{ctg} \theta_{0}\left(\alpha_{v}+1\right)}{2 \varphi_{1} \sin \theta_{0}}\left(w_{b_{2}}-w_{b_{1}}\right)+ \\
& +\frac{\alpha \beta_{v}}{4 \varphi_{1} \theta_{0} \sin \theta_{0}}\left(w_{b_{5}}-w_{b_{6}}-w_{b_{7}}+w_{b_{8}}\right)=0 \\
& \frac{1}{2} \frac{d^{2} \omega^{2}}{d t^{2}}-\frac{d \omega_{b}}{d t}-\frac{1}{\sin ^{2} \theta_{0}} \omega_{b}+\frac{\alpha \rho_{v} c t g \theta_{0}}{2 \theta_{0}}\left(\omega_{b_{4}}-\omega_{b_{3}}\right)+\frac{1}{2 \varphi_{1}^{2} \sin ^{2} \theta_{0}}- \\
& -\left(2 \omega_{b}+\omega_{b_{1}}+\omega_{b_{2}}\right)+\frac{\alpha_{v} \alpha^{2}}{\theta_{0}{ }^{2}}\left(-2 \omega_{b}+\omega_{b_{3}}+\omega_{b_{4}}\right)-\frac{3 \operatorname{ctg} \theta_{0}}{2 \varphi_{1} \sin \theta_{0}}\left(v_{b_{2}}-v_{b_{1}}\right)+ \\
& +\frac{\alpha \beta_{v}}{4 \varphi_{1} \theta_{0} \sin \theta_{0}}\left(v_{b_{3}}-v_{b 6}-v_{b_{7}}+v_{b_{8}}\right)- \\
& -\operatorname{ctg} \theta_{0} u_{b}+\operatorname{ctg} \theta_{0} \frac{d u_{b}}{d t}+\frac{\alpha \alpha_{v}}{\theta_{0}}\left(u_{b_{4}}-u_{b_{3}}\right)+\frac{\alpha \beta_{v}}{2 \theta_{0}} \frac{d}{d t}\left(u_{b_{4}}-u_{b_{3}}\right)=0
\end{aligned}
$$

Omitting the intermediate computations, we give the final stress expressions for the edge $m$, compiled in finite differences with respect to the variables $\theta$ and $\varphi$. Equations of pressure for a compound body of a structure consisting of a cylindrical body and a sphere: 


$$
\begin{aligned}
& \sigma_{r}=\frac{E}{1+v}\left[\begin{array}{l}
2 \rho_{v} \frac{u_{b}}{r_{0}}+\alpha_{v} \frac{d u_{b}}{d r}+\frac{\rho_{v}}{2 r_{0} \varphi_{1} \sin \theta_{0}}\left(v_{b_{2}}-v_{b_{1}}\right)+ \\
\frac{\rho_{v} \operatorname{ctg} \theta_{0}}{r_{0}} \omega_{b}+\frac{\alpha \rho_{v}}{2 r_{0} \theta_{0}}\left(\omega_{b_{4}}-\omega_{b}\right)
\end{array}\right] ; \\
& \sigma_{\varphi}=\frac{E}{1+v}\left[\begin{array}{l}
2 \beta_{v} \frac{u_{b}}{r_{0}}+\rho_{v} \frac{d u_{b}}{d r}+\frac{\alpha_{v}}{2 r_{0} \varphi_{1} \sin \theta_{0}}\left(v_{b_{2}}-v_{b_{1}}\right)+ \\
\frac{\alpha_{v} \operatorname{ctg} \theta_{0}}{r_{0}} \omega_{b}+\frac{\alpha \rho_{v}}{2 r_{0} \theta_{0}}\left(\omega_{b_{4}}-\omega_{b_{3}}\right)
\end{array}\right] \\
& \sigma_{\theta}=\frac{E}{1+v}\left[\begin{array}{l}
2 \beta_{v} \frac{u_{b}}{r_{0}}+\rho_{v} \frac{d u_{b}}{d r}+\frac{\rho_{v}}{2 r_{0} \varphi_{1} \sin \theta_{0}}\left(v_{b_{2}}-v_{b_{1}}\right)+ \\
\frac{\rho_{v} \operatorname{ctg} \theta_{0}}{r_{0}} \omega_{b}+\frac{\alpha \alpha_{v}}{2 r_{0} \theta_{0}}\left(\omega_{b_{4}}-\omega_{b}\right)
\end{array}\right] ; \\
& \tau_{r_{\varphi}}=\frac{E}{2(1+v)}\left[\frac{1}{2 r_{0} \varphi_{1} \sin \theta_{0}}\left(u_{b_{2}}-u_{b_{3}}\right)+\frac{d v_{b}}{d r}-\frac{v_{b}}{r_{0}}\right] \\
& \tau_{\varphi \theta}=\frac{E}{2(1+v)}\left[\frac{\alpha}{2 r_{0} \theta_{0}}\left(v_{b 4}-v_{b_{3}}\right)-\frac{\operatorname{ctg} \theta_{0}}{r_{0}} v_{b}+\frac{1}{2 r_{0} \varphi_{1} \sin \theta_{0}}\left(w_{b_{2}}-w_{b_{1}}\right)\right] \\
& \tau_{\theta r}=\frac{E}{2(1+v)}\left[\frac{d w_{b}}{d r}-\frac{w_{b}}{r_{0}}+\frac{\alpha}{2 r_{0} \theta_{0}}\left(u_{b_{4}}-u_{b_{3}}\right)\right]
\end{aligned}
$$

\section{Results}

Using the software complex, which facilitates the calculation of the mathematical apparatus for the derived stress tensor, we obtain the following values, shown in Fig. 4.

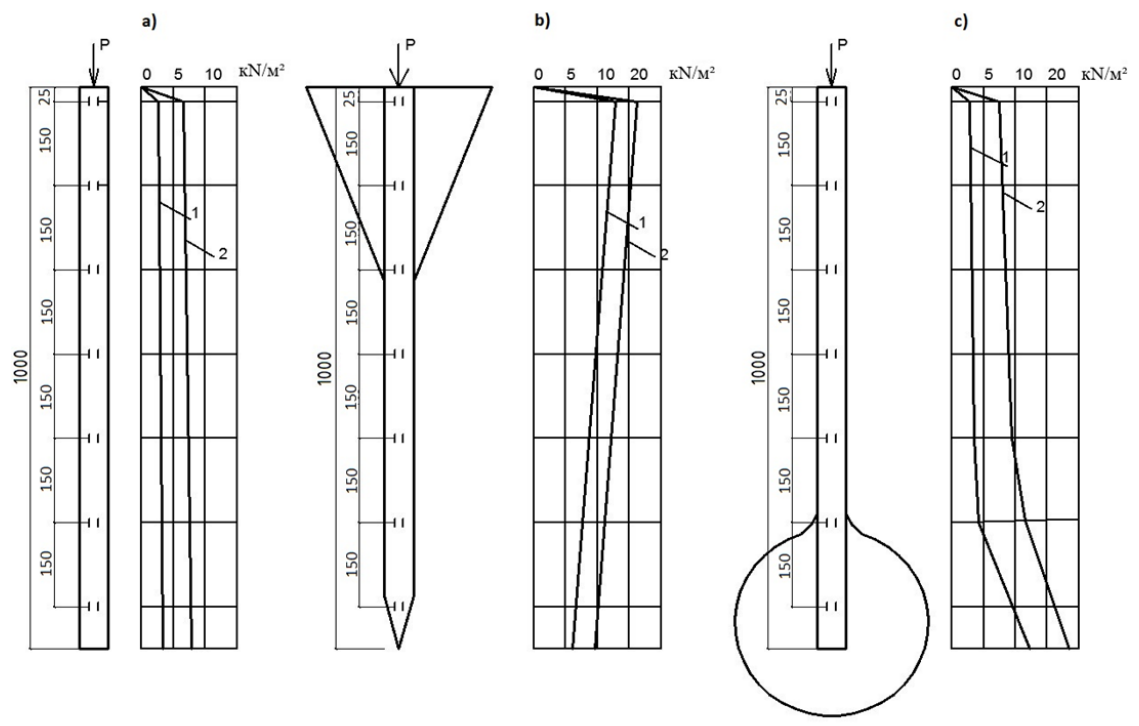

Fig. 4. Diagrams of normal and tangential pressure for three types of piles: A - usual prismatic, Bprismatic with a broadening in the form of prefabricated wedges at the top, $\mathrm{V}$ - with broadening at the bottom 


\section{Conclusion}

The determination of pressure in the near-horizon space of a compounded array in the form of a pile structure with broadening in solving a problem with the formulation of differential equations in a discrete method in rectangular and spherical coordinates is a cumbersome and complex mathematical apparatus for a modern design engineer. This method was developed in the early 50's of last century. At present, as the analysis has shown, the solution of complex geotechnical problems of soil bases and foundations under various types of loads in numerical modeling is carried out with the help of modern software: MIDAS GTS NX, FEMAP NE/NASTRAN, COSMOS, ЛИPA, FEM/MODELS, SCAD, ANSYS, Z/SOIL, URAN, PLAXIS and others, which allow to pass with high efficiency to the analysis of massive three-dimensional bodies and their fragments of completely arbitrary and complex shape [2-7].

\section{References}

1. L. P. Vinokurov, Direct methods for solving spatial and contact problems for foundations and massifs (Kharkov, 1956)

2. V. S. Fedorov, N. V. Kupchikova, Bulletin of civil engineers, 1 (2011)

3. S. B. Kositsyn, V. S. Fedorov, V. Y. Akulich, Building Construction and Architecture, 1 (2018)

4. N. V. Kupchikova, Bulletin of civil engineers, 1 (2015)

5. V. M. Bondarenko, V. S. Fedorov, AISI, 8 (2014)

6. E. N. Kurbatskii, Guidelines for solving problems of mechanics using Fourier transformation (Institute of communications, Moscow 1979)

7. E. N. Kurbatskii, Construction of residential buildings, 4 (1987) 